\title{
Assessment of Serum Tryptase Activity Among Acute and Chronic Myeloid Leukemia Patients Visiting Hematology-Oncology Clinic at TikurAnbessa Specialized Hospital, and Comparison with Healthy Controls
}

\author{
Endriyas Kelta ${ }^{1}$, Frank Ashall ${ }^{2}$, Abdulaziz Abubeker ${ }^{3}$ \\ ${ }^{1}$ Department of Biomedical Sciences, Jimma University, Jimma, Ethiopia \\ ${ }^{2}$ Department of Biochemistry, Addis Ababa University, Addis Ababa, Ethiopia \\ ${ }^{3}$ Department of Internal Medicine, Addis Ababa University, Addis Ababa, Ethiopia
}

Email address:

keltatona@gmail.com (E. Kelta), frankashall@yahoo.com (F. Ashall), abdulazizas88@gmail.com (A. Abubeker)

To cite this article:

Endriyas Kelta, Frank Ashall, Abdulaziz Abubeker. Assessment of Serum Tryptase Activity Among Acute and Chronic Myeloid Leukemia Patients Visiting Hematology-Oncology Clinic at TikurAnbessa Specialized Hospital, and Comparison with Healthy Controls. Cancer Research Journal. Vol. 6, No. 1, 2018, pp. 26-37. doi: 10.11648/j.crj.20180601.15

Received: January 3, 2018; Accepted: January 29, 2018; Published: March 5, 2018

\begin{abstract}
Tryptase is a serine protease that is expressed in leukemic cells of chronic myeloid leukemia (CML) patients and in blasts of acute myeloid leukemia (AML) patients. Tryptase may be useful for diagnosis, assessment of severity of disease (leukemic cell burden), monitoring minimal residual disease and prognosis of AML and CML patients. The main objective of this study was to assess the serum levels of tryptase activity among CML and AML patients and to compare the serum levels of tryptase activity of acute and chronic myeloid leukemia patients with each other and with those of healthy controls. To meet this objective, a hospital-based comparative cross-sectional study was conducted among CML and AML patients from February 2016 up to December 2016. Serum samples were obtained from 24 AML, 60 CML and 35 healthy controls. Fluorogenic assays for serum tryptase activity using aminomethylcoumarin (AMC) peptide derivative were carried out. Statistical analysis was done by using SPSS version 20. Descriptive statistics, Paired Samples T-test, Wilcoxon Signed Rank test and Spearman's rho test were used to investigate any correlation among different parameters. The minimum level of statistical significance was set at $p$-value $<0.05$. Accordingly, the mean and median serum levels of tryptase activity were significantly higher in patients with AML and CML than in the healthy controls (P-value $<0.05$ ). CML patients in chronic phase (CP) and secondary AML patients had significantly higher mean and median serum levels of tryptase activity than CML patients in accelerated/blast phase (AP/BP) and de novo AML patients ( $\mathrm{p}$-value $<0.05)$. These elevated mean and median levels of serum tryptase activities were due to a subset of individuals with elevated serum tryptase levels (41.7\% of AML \& $30 \%$ of CML); the remaining leukemic individuals (58.3 \% AML \& $70 \%$ of CML) had normal serum levels of tryptase activity. Finally, it was concluded that the serum tryptase level might be a useful diagnostic and prognostic marker in a subset of patients with CML and AML. However, further studies that incorporate other protocols such as tryptase immunoassay are warranted to exclude contaminant non-tryptase proteases from the serum samples.
\end{abstract}

Keywords: CML, AML, Serum Tryptase Activity

\section{Introduction}

\subsection{Biochemistry of Human Tryptase}

Tryptase is a $134 \mathrm{kDa}$ neutral serine protease [3], [11] which is highly expressed in mast cells and to a lesser extent in peripheral blood basophils [10], [24], and also in leukemic cells of CML patients [25] and in blasts of AML patients [26]. Peripheral blood basophils express 500-fold less tryptase than mast cells in tissue [28]. 
Tryptase derives its name from its characteristic trypsin-like substrate specificity in which it cleaves peptide or protein substrates preferentially on the carboxyl side of lysine $(\mathrm{K})$ or arginine (R) residues [3], [24]. Like many other proteases, tryptase cleaves a scissile peptide bond in a protein or peptide or amide bond in a synthetic peptidyl chromogenic or fluorigenic substrate. Substrate amino acids that are on the amino (N) side of the scissile bond are numbered P1, P2, and $\mathrm{P} 3$, and so on (called P sites) with the one closest to the scissile bond numbered $\mathrm{P} 1$, and those that are on the carboxyl (C) side of the scissile bond are numbered as P1', P2', P3' and so on (called $\mathrm{P}^{\prime}$ sites). Their corresponding binding sites on the protease are labeled with $\mathrm{S}(\mathrm{S} 1, \mathrm{~S} 2$, and $\mathrm{S} 3 \ldots)$ and $\mathrm{S}^{\prime}$ (S1', S2' and $\mathrm{S} 3$ '..., respectively (as shown in Figure 1) [30], [31].
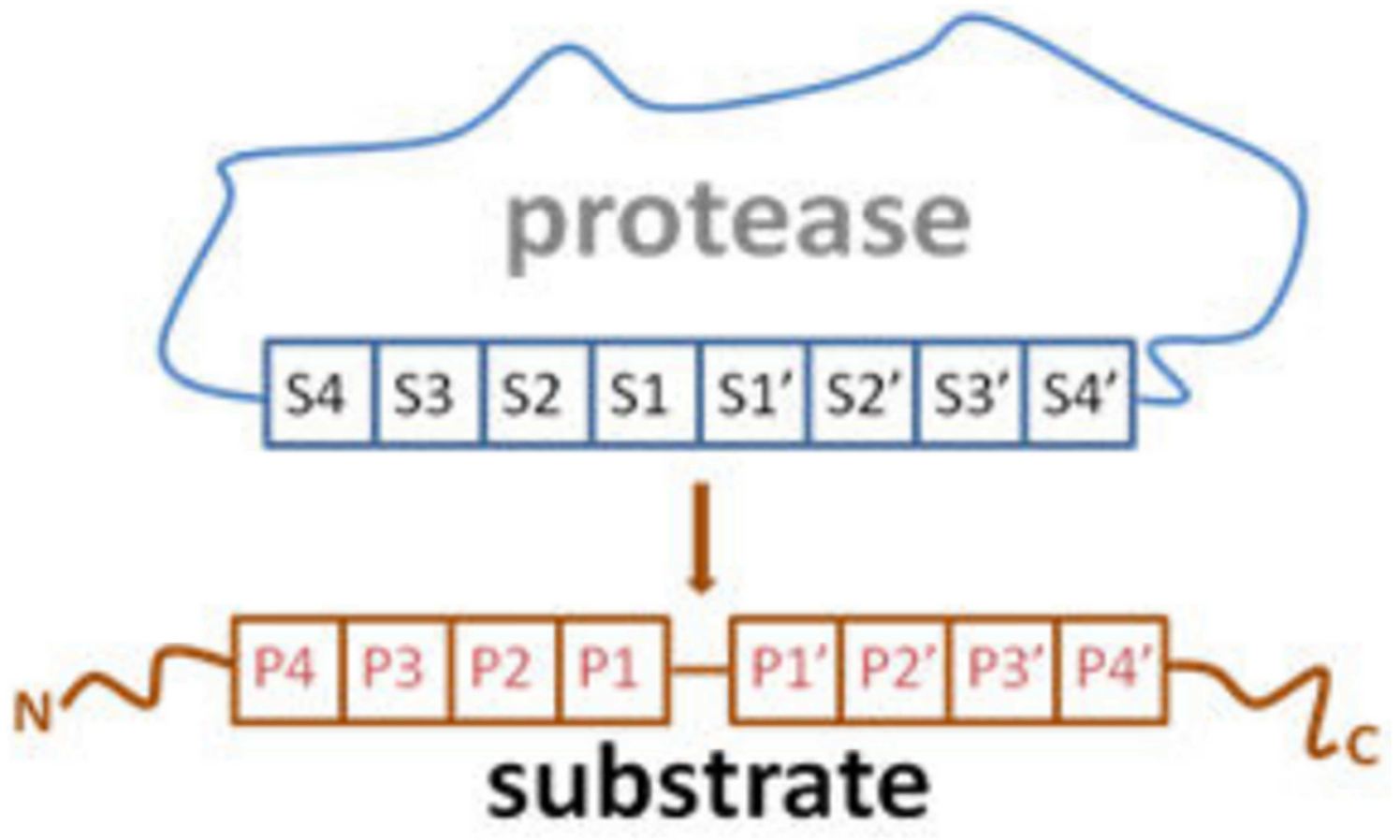

Figure 1. General nomenclature for protease reactions [31].

\subsection{Tryptase as a Therapeutic Target in Cancer}

Normally, stem cell factor (SCF) binds to tyrosine kinase c-KitR on the surface of tryptase -positive human mast cells and activates mast cells. The activated mast cells then, degranulate and release tryptase, an agonist of one of the Gprotein coupled receptor super-family, proteinase-activated receptor-2 (PAR-2) of epithelial and endothelial cells, which directly activates PAR-2.The activated PAR-2 results in the cleavage of the N-terminal extracellular portion of PAR-2, exposing the new $\mathrm{N}$-terminal sequence, which in turn can bind to a yet undefined binding site of the G-protein coupled receptor, leading to G-protein coupled signal transduction that results in phosphatidylinositol hydrolysis and elevation of $\left(\mathrm{Ca}^{2+}\right)$ in numerous types of cells including tumor cells, and which in turn, lead to tumor cell angiogenesis and proliferation[10], [15], [16].

For instance, the proliferation signal induced by tryptase on human colon carcinoma cell lines after PAR-2 activation leads to the increase in calcium level via G- protein coupled signal transduction and short time phosphorylation of mitogen-activated protein kinase/extracellular signal-related kinase (MEKK) and the mitogen-activated protein kinase (MAPK) pathway. The increase in calcium level via Gprotein coupled signal transduction also led to the activation of Cyclo-oxygenase-2 (COX-2) and prostaglandin $\mathrm{E}_{2}\left(\mathrm{PGE}_{2}\right)$ synthesis. Together, the MEKK and MAPK pathway activation and $\mathrm{PGE}_{2}$ synthesis were essential for human colon carcinoma cell line proliferation (Figure 2)[15].

In addition, a cytoplasmic adaptor protein, Sodiumhydrogen antiporter-3 regulator-1 (NHERF-1), present in various cell types including tumor cells, regulates so manytransmembrane receptors, transporters and other proteins located near the plasma membrane, and through the Ezrin/protein kinase-A- mediated means, leads to cancer progression (Figure 2). Besides these, PAR-2 activation by tryptase increases the VEGF expression, on endothelial and intestinal cell surfaces, which in turn, stimulates VEGFR-2 positive tumor, mast and endothelial cells directly and results in the tumor growth and angiogenesis[14].

Therefore, the correlation between tryptase released from mast cells as pro-angogenic factor and tumor angiogenesis and progression could suggest the role of human mast cells and tryptase as therapeutic targets in cancer.Particularly, by hindering mast cell activation and degranulation via c-KitR tyrosine kinase inhibitors such as imatinib and masitinib and by inhibiting the release of tryptase from mast cells via tryptase inhibitors such as gabexate and nafamostat mesylate, it is possible to inhibit tumor cell angiogenesis, growth, invasion and metastasis (as shown in Figure 2)[15]. 


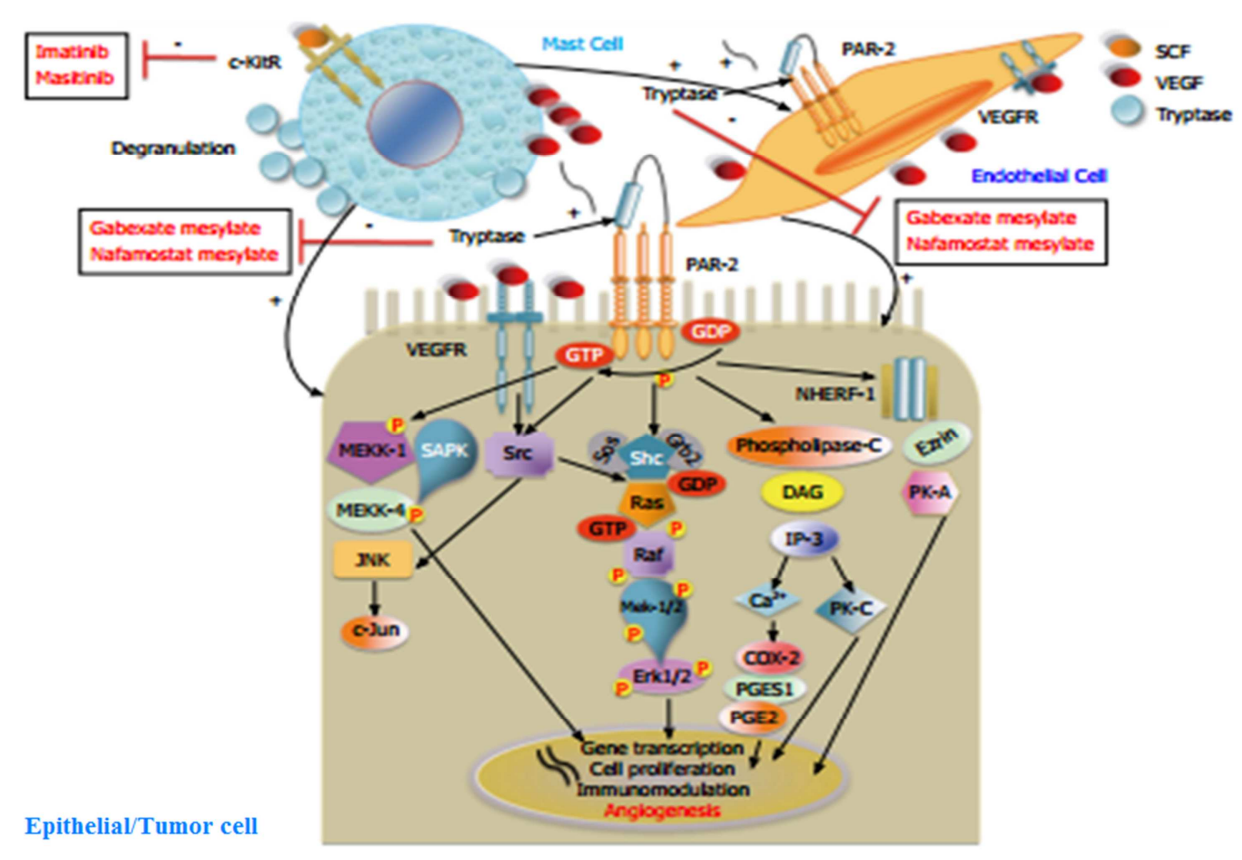

Figure 2. Human mast cells and their tryptase as therapeutic target in cancer [15].

\subsection{Tryptase in Leukemia}

Leukemia is a disease of blood or bone marrow characterized by the rise in number of abnormal leukocytes or white blood cells (WBC). The abnormality in leukemic leukocytes is manifested due to inhibition of normal differentiation of progenitor cells and/or increased proliferation rate of leukocytes[7]. Unlike normal blood cells, leukemia cells do not die when they become old or damaged and can build up and crowd out normal blood cells thereby, lower the level of normal blood cells and make it harder for the body to get oxygen to the tissues, control bleeding, or fight infections[1], [20].

Leukemia is classified into acute and chronic based on whether most of the leukemic cells resemble normal mature leukocytes or immature hematopoietic stem cells, and lymphocytic and myeloid based on the types of bone marrow cells that are affected [1], [7].

Accordingly, acute leukemia cells are immature blood cells, also called blasts. Like normal blast cells, these cells divide quickly, and unlike normal blast cells do not stop dividing when normal blast cells stop dividing.Chronic leukemia cells, on the other hand, are more mature cells and unlike normal leukocytes, are not completely matured, generally do not fight infections, survive longer, buildup and crowd out normal cells[1], [20]. Considering the type of bone marrow cells that are influenced, myeloid leukemia begins in immature forms of myeloid lineages, that give rise to erythrocytes, megakaryocytes, and leukocytes other than lymphocytes whereas, lymphocytic leukemia starts in immature forms of lymphoid lineages that are mainly leading to lymphocytes [1].

By amalgamating both of leukemia classification criterion, that is, maturity of leukemic cells and bone marrow cell types that are affected, there are four main types of leukemia. These are: Acute myeloid leukemia (AML), Chronic myeloid leukemia (CML), Acute lymphocytic leukemia (ALL), and Chronic lymphocytic leukemia (CLL) [1], [7], [20].

Acute myeloid leukemia (AML) is a serious hematopoietic cell cancer characterized by continuous proliferation and accumulation of immature myeloid blast cells[25]. Based on the type of bone marrow cell from which the leukemia developed, and on its degree of maturity, before 2001, in the 1970s the French-American-British (FAB) leukemia experts divided AML into nine subtypes, (M0-M7 and M4eos). M0M7 FAB subtypes indicate undifferentiated AML, AML with minimal maturation, and AML with maturation, acute promyelocytic leukemia (APL), acute myelomonocytic leukemia, acute monocytic leukemia, acute erythroid leukemia, and acute megakaryoblastic leukemia respectively, and (M4eos) indicates acute myelomonocytic leukemia with eosinophilia [1], [7].

One drawback of FAB classification system is that it does not incorporate factors that are currently known to affect prognosis of AML patients. To solve this limitation, WHO in 2001 introduced a more precise division that comprises cytogenetic abnormalities and prognostic significance, and revised in 2008 and classified AML in to four classes, M0M3. These are: AML with recurrent genetic abnormalities, AML with myelodysplasia-related changes, therapy-related myeloid neoplasms, and AML not otherwise specified, respectively [1], [7].

In distinct versions of AML, features of chromosomal abnormalities, such as translocation between chromosomes 8 and 21, inversion of chromosome 16 and translocation between chromosomes 15 and 17, are suggestive of a favorable prognosis; and other cytogenetic features, like deletion of part of chromosome 5 or 7 , translocation or 
inversion of chromosome 3, translocation between chromosomes 6 and 9, translocation between chromosomes 9 and 22 ,abnormalities of chromosome 11 , and other complex changes of several chromosomes,are indicative of unfavorable or poor prognosis. Some of poor prognostic signs in AML patients, therefore, are a high load of leukemic cells, unfavorable chromosomal abnormalities, a poor response to induction chemotherapy, and minimal residual disease or recurrent AML[1], [25].

Chronic myeloid leukemia (CML) is a cancer of blood cells, characterized by replacement of the bone marrow with malignant, leukemic cells.CML is usually diagnosed by finding a specific chromosomal abnormality called the Philadelphia $(\mathrm{Ph})$ chromosome. In hematopoietic (blood making) cells of bone marrow abl-gene on chromosome 9 undergoes reciprocal translocation (shifts) to bcr-gene on chromosome 22 , fuses with bcr-gene, and makes mRNA which has some complementary area with bcr-gene and another complementary area with abl-gene. This mRNA will be translated into pathological proteins or chimeric proteins, also called BCR-ABL proteins (as shown in Figure 5), which give cell signals for over growth and proliferation, and the risk of cancer will be increased. This abbreviated chromosome 22 which lost its large portion to chromosome 9 and got a very small portion from chromosome 9, and eventually develops bcr-abl gene fusion, is called Philadelphia chromosome [17].

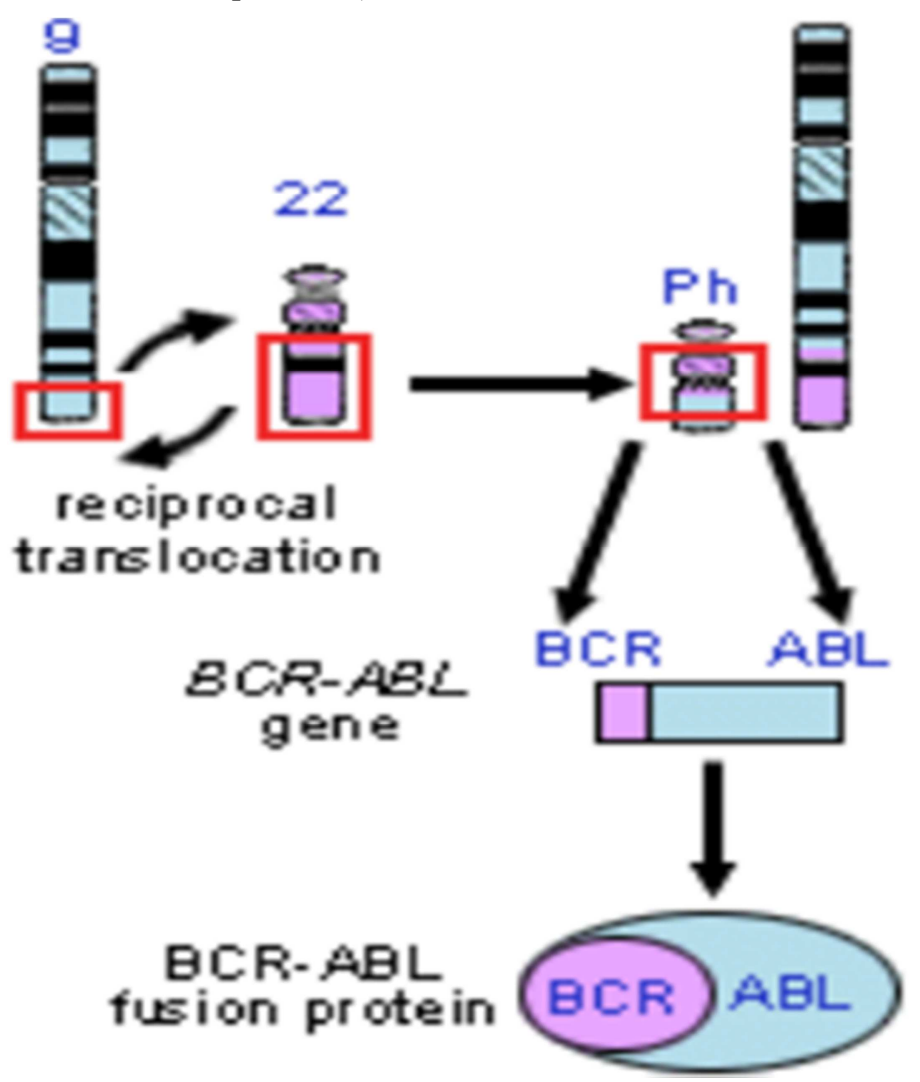

Figure 3. Formation of BCR-ABL chimeric protein by reciprocal translocation between abl-gene on chromosome 9 and bcr-gene on chromosome-22 [17].

Over $90 \%$ of CML patients carry this chromosomal abnormality, and the drugs that selectively work against the tyrosine kinase activity of the fusion protein BCR-ABL, should be toxic mainly to CML malignant cells[7]. In majority ofpatients with $\mathrm{CML}$, the $\mathrm{BCR} / \mathrm{ABL}$ tyrosine kinase inhibitors, like imatinib, serve as the golden standard of firstline treatment and produce major cytogenetic and molecular responses and have considerably improved the prognosis[26]. For example, the introduction of imatinib and other similar specific inhibitors of BCR-ABL have increased rates of complete hematological response to $97 \%$ and complete cytogenetic response to $85 \%$. It was stated in some other studies that the rate of complete cytogenetic response increased from $30 \%$ with interferon-alpha to more than $70 \%$ with imatinib[7], [26]. In a number of patients, on the other hand, these first generation tyrosine kinase inhibitors fail to provide a durable major cytogenic and molecular response. For these patients and others (for young and fit patients), second- or third generation tyrosine kinase inhibitors as well as stem cell transplantation (SCT) has to be considered. However, SCT is linked with substantial morbidity and mortality, and second- or third generation tyrosine kinase inhibitors have overwhelming side effects [26].

In addition to Philadelphia ( $\mathrm{Ph}$ ) chromosome used in diagnosis of CML, several markers are available currently, for diagnosis of CML and AML. Some of such diagnostic markers include PCR- measuring of breakpoint-related gene products, flow cytometric determinations and or cytogenetic measurements. All these markers have some potential drawbacks namely, less feasibility, less sensitivity and 
constantly being positive regardless of long-term survival and continued remission [25].

Measurement of serum tryptase levels may therefore be used as a marker of major clinical importance and might be helpful in designing treatment options like further aggressive therapy, maintenance therapy and or stem cell transplantation in patients with CML and recurrent AML. Tryptase may also be useful for diagnosis, assessment of severity of disease (leukemic cell burden), monitoring minimal residual disease and prognosis of both AML and CML patients. These statements are consistent with recent findings suggesting the production of the significant amount of tryptase by the AML blasts in patients with AML and immature CML basophils in patients with CML. In both cases, the serum tryptase levels were elevated and became the potential marker of leukemic cell burden (AML blast load in AML and CML basophil load in CML) [25], [26].

Data recently suggest that tryptase is selectively expressed in neoplastic cells of myeloid leukemia than those of lymphoid leukemia. In one study, which aimed to determine the serum tryptase levels of 914 patients with hematological malignancies, the elevated serum tryptase levels, defined as greater than $15 \mathrm{ng} / \mathrm{ml}$ [13], seen in almost all patients of myeloid neoplasm, while almost all patients of lymphoid neoplasm exhibit normal serum tryptase levels, defined as less than or equals to $11.4 \mathrm{ng} / \mathrm{ml}$ [13]. In the same study, from the total of 317 AML and $156 \mathrm{CML}$ patients, $38 \%$ and $34 \%$ respectively, experienced an elevated serum tryptase level [27]. In one other study that was aimed to determine the serum total tryptase levels of 108 patients with de novo AML and 25 patients with secondary AML by fluorescence immunoassay, the elevated serum tryptase levels, defined as greater than $15 \mathrm{ng} / \mathrm{ml}$ [13], were detected in 39\% of patients with de novo AML and $44 \%$ of those with secondary AML [28]. In another study that aimed in determining the serum total tryptase levels in 168 de novo AML patients, the elevated serum tryptase levels were seen in $36.3 \%$ of patients with de novo AML. Likewise, the serum total tryptase levels were elevated in $45 \%$ of 155 de novo AML patients screened for the study aimed in checking serum total tryptase levels at diagnosis of AML as a simple tool for selecting patients to KIT mutations [4].

It has also been stated that in CML patients, as immature CML basophiles synthesize and release significant quantities of tryptases, tryptase may become potential marker of the total basophil load. It has also been shown that patients with advanced (accelerated and blast) phase CML more often possess elevated serum-tryptase levels than patients with Chronic Phase CML. In addition, in CML patients with elevated serum tryptase levels, there is increase in BCR-ABL protein expression compared to those with normal serum tryptase levels [26].

It was also shown recently that serum tryptase levels correlate with poor prognostic signs before chemotherapy, and return to normal or near normal values in patients achieving a hematologic clinical remission after induction chemotherapy[25]. In the one of study mentioned so far, that determine the serum total tryptase levels of 108 patients with de novo AML and 25 patients with secondary AML by fluorescence immunoassay, the serum total tryptase levels of de novo and secondary AML patients elevated before chemotherapy were returned to normal or near normal values, defined as less than/equals to $11.4 \mathrm{ng} / \mathrm{ml}$, in de novo and secondary AML patients who entered complete remission after chemotherapy [28]. Similarly, in the study that determine the serum total tryptase levels in 168 de novo AML patients, the serum total tryptase levels of de novo AML patients elevated before chemotherapy were returned to normal or near normal values in $69 \%$ of de novo AML patients who entered complete hematological remission afterinduction chemotherapy[25]. Serum tryptase level at diagnosis, therefore, may serve as a new biomarker improving prognosis in leukemia patients mainly, CML and recurrent AML [25], [26].

\subsection{Stetment ofthe Problem}

Most of the current reports on leukemia emerge from countries with well established science capacities [9]. In the United States, for instance; approximately, 43,050 individuals (24,690 men and 21,840 women) were diagnosed with leukemia, and 21,840 died of the disease in the year in 2010. Thirty-three percent of cancers in children and 1340 deaths yearly were accounted by leukemia and so, leukemia is often thought of as a disease of childhood. In children, the highest incidence is in between 1 to 4 and the highest death rate is in between 10 and 19, even the incidence is 10 times higher in adults. The median age for diagnosis is 66, and median age of death from leukemia is 74 [14].Similarly, in Europe, approximately 18,000 new patients were diagnosed with leukemia each year representing approximately, $0.6 \%$ of all cancers. AML presented with a median age at presentation of 64 years, accounting for around $30 \%$ of all leukemia in adults. CML presented with a median age at presentation around age 60.CML comprised only around $2 \%-3 \%$ of all the leukemia diagnosed in patients $<20$ years of age [9].

In contrast, as may be due to the deficiency of statistics or failure of diagnosis, the lowest rates of leukemia reported in sub-Saharan Africa, including Ethiopia. An early report from Uganda on leukemia among African children in Uganda, suggested a very low prevalence of leukemia [6], [9]. A study of pediatric leukemia in Cameroon showed that AML comprised $21.4 \%$ of all pediatric acute leukemia [9], [19]. In Kenya, leukemia in children below the age of 15 years comprised $37 \%$ of leukemia in all ages. AML comprised of $42 \%$ all pediatric acute leukemia [9], [12]. A recent study in south Nigeria showed that AML comprised $12.3 \%$ of all leukemia with a mean age of diagnosis of 25.6 years, and CML comprised of $23.9 \%$ of all leukemia, with a mean age at diagnosis of 35.2 years [9], [18]. In Ethiopia, a report showed that the commonest type of leukemia was CML followed by ALL and AML [9], [22].

The disparity between developed and developing 
countries, including Ethiopia, may be due to failure of proper diagnostic system and may be the reflection of the capacity of individual countries or research groups to choose more feasible and sensitive markers for leukemia diagnosis, particularly AML and CML [9].

\subsection{Significance of the Study}

This study focused on serum level of tryptase activity thatmay solve the problems of feasibility, sensitivity and constant positivity related to the potential diagnostic markers of AML and CML used now a day. It might also be helpful in designing treatment options such as further aggressive therapy, maintenance therapy and or stem cell transplantation in patients with recurrent AML and 'high risk CML patients'.

In addition, no study done so far on the serum levels of tryptase activity of CML/AML patients in Ethiopia, but the elevated serum tryptase level seen elsewhere may become a new marker in the diagnosis of CML/AML. Thus, in this study it is essential to evaluate the magnitude of serum tryptase of AML/CML patients in order to determine serum tryptase level as a diagnostic criterion of $\mathrm{AML} / \mathrm{CML}$ in Ethiopia. The findings of the present study could show the importance of routine measurement of serum tryptase levels for diagnosis of CML/AML patients and could also be a stepping stone for future large scale prospective studies in the country.

\subsection{Objectives of the Study}

\subsubsection{General Objectives}

The main objective of this study was to assess the serum levels of tryptase activity among chronic and acute myeloid leukemia patients and to compare the serum levels of tryptase activity of acute and chronic myeloid leukemia patients with each other's and with those of healthy individuals.

\subsubsection{Specific Objectives}

a. To assess the levels of tryptase activity in serum from CML, AML and healthy individuals.

b. To compare serum levels of tryptase activityof $\mathrm{CML}$ patients with those of AML patients.

c. To compare serum levels of tryptase activity of AML and CML patients with those of healthy individuals.

\section{Materials and Methods}

\subsection{Study area and Period}

The study was conducted in the Department of Biochemistry, College of Health Sciences, Addis Ababa University in collaboration with Hematology-Oncology clinic, Tikur Anbessa Specialized Hospital, Addis Ababa, Ethiopia from February, 2016 to December, 2016.

Tikur Anbessa Specialized Hospital, located in the nation's capital Addis Ababa, is Ethiopia's largest referral hospital in the country. The hospital was given to Addis Ababa University by the Ministry of Health as a main teaching hospital in school of medicine. TASH offers diagnosis and treatment for approximately 370,000 to 400,000 patients a year. The hospital has 600 beds ( 18 beds dedicated for cancer care), with 16 specialists, 50 non-teaching physicians.

\subsection{Study Design}

A hospital based comparative cross-sectional study was conducted to achieve the objectives of the study.

\subsection{Population}

\subsubsection{Source Population}

All adult CML and AML patients visiting HematologyOncology Clinic at TASH, Addis Ababa, Ethiopia and all presumed healthy staff members of Kolfe Keraniyo Sub-city Lome Meda Health Center.

\subsubsection{Study Population}

During the study period 119 individuals between 18 and 75 years old, including 65 males and 54 females, were studied. AML and CML patients were recruited from the Hematology-Oncology Clinic at Tikur Anbessa Hospital. The study participants were organized into the following study groups: one group consisted of 24 AML patients, ranging from 19 to 75 years old; another group consisted of $60 \mathrm{CML}$ patients, ranging from 18 to 70 years old; the third group consisted of 35 healthy controls, ranging from 26 to 66 years old. Presumed healthy controls were randomly selected individuals from the staff of Kolfe Keranyo sub-city Woreda13 Lomi Meda Health Center who were presumed healthy. They had no known chronic diseases and were not taking any chronic medications.

\subsubsection{Exclusion and Inclusion Criteria}

AML/CML patients with a previous history of allergic airway diseases (e.g. asthma), rheumatoid arthritis, mastocytosis, hyper-eosinophilia and anaphylactoid reactions (e.g. skin rashes, blushing and urticaria) were excluded from the study (as the serum tryptase levels are elevated abnormally under these conditions).

\subsection{Sampling Procedure}

CML and AML patients who were able to comply with this study and volunteered to be involved in the study were recruited during the specified study period. Due to the restrictions on time and cost as well as availability of patients, only 84 morphologically confirmed leukemia (60 CML and 24 AML) patients and 35 healthy control individuals were recruited.

\subsection{Study Variables}

\subsubsection{Dependent Variables}

a. Serum levels of tryptase activity

\subsubsection{Independent Variables}
a. Age
b. Sex
c. Classes of AML: de novo (primary) versus secondary
d. Stages of CML: chronic versus accelerated/blast stage 


\subsection{Ethical Considerations}

After being approved by the Research and Ethics Committee of the Department of Biochemistry, School of Medicine, College of Health Sciences, Addis Ababa University, a formal letter was written to HematologyOncology clinic at TASH. Similarly, the objectives of the study were explained to all concerned bodies assigned in the hospital to get permission and support. Also the aim of the study was clearly stated for the participants (both patients and controls) under the study. Moreover, confidentiality was strictly maintained throughout the course of the study and the study outcome was submitted to the hospital as well.

\subsection{Serum Sample Preparation and Serum Tryptase Activity Assay Protocols}

\subsubsection{Serum Sample Preparation}

About $5 \mathrm{~mL}$ of blood was drawn from the antecubital vein of patients and healthy controls and then left to clot at room temperature for one hour. The blood was centrifuged at 3000 rpm for 10 minutes and the serum removed and stored at minus 70 degrees centigrade until use.

\subsubsection{Serum Tryptase Activity Assay Protocol}

Principles: Tryptase cleaves a scissile peptide bond in a protein or peptide or an amide bond in a synthetic peptidyl chromogenic or fluorigenic substrate. Substrate amino acids that are on the amino $(\mathrm{N})$ side of the scissile bond are numbered P1, P2, and P3, and so on (called P sites) with the one closest to the scissile bond numbered $\mathrm{P} 1$, and those that are on the carboxyl (C) side of the scissile bond are numbered as P1', P2', P3' and so on (called P' sites). Their corresponding binding sites on the protease (tryptase) are labeled with S (S1, S2, and S3 ...) and S' (S1', S2' and S3'..., respectively (as shown in Figure 1) [30], [31].

In this study a fluorogenic assay protocol was used in which a fluorescent dye, 7-amino-4-methylcoumarin (AMC) that contains a reactive amine group covalently attached to the carboxyl end of a flourogenic substrate (Ac-Orn-PheArg-AMC) via an amide bond. The sequence of the peptide moiety provides tryptase specificity and the fluorescent AMC dye moiety functions as a fluorogenic reporter for tryptase activity. Trptase cleaves the amide bond, which mimics a peptide bond, on the carboxyl side of arginine. At the excitation and emission wavelengths used, the fluorescence of the attached AMC is quenched when it is covalently attached to the peptide via an amide bond. At these wavelengths, the fluorescence increases when AMC is released by tryptase cleavage of the amide bond that joins the AMC to the peptide moiety (Figure 4) [31].

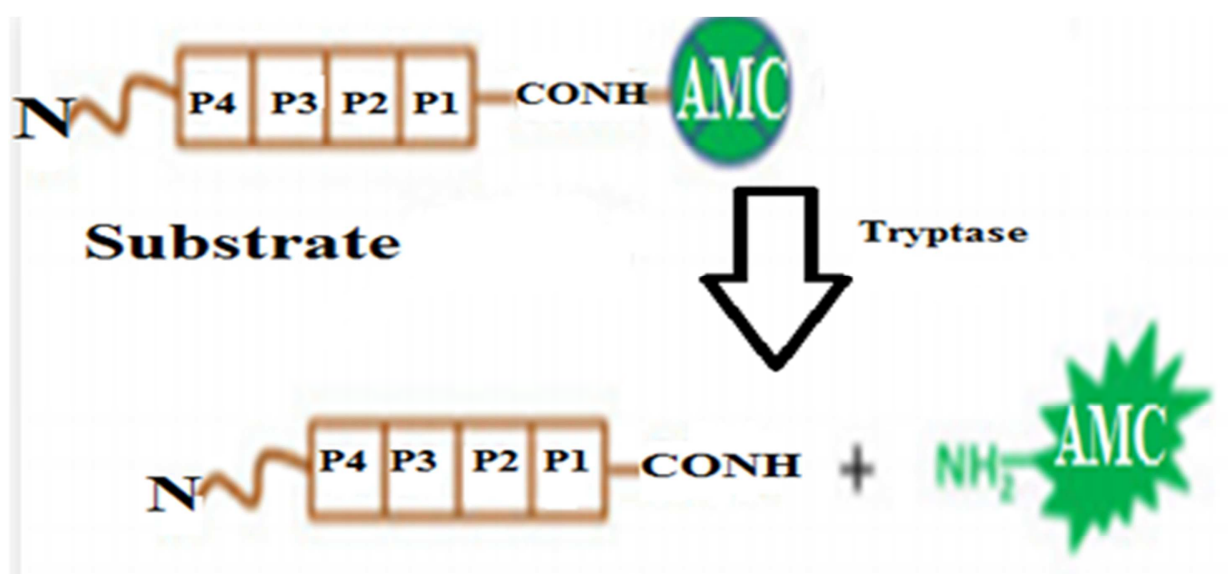

\section{Products}

Figure 4. Basic principles of fluorescence-based assay using peptidyl-AMC substrates for measuring proteolytic activity of Tryptase, through release of free $A M C[31]$.

Tryptase within the serum samples cleaves the substrate; $\mathrm{N}$-Acetyl-Orn-Phe-Arg-AMC specifically on the C-terminal side of the arginyl residue and aminomethylcoumarin(AMC) is released as a product. AMC released was detected using an excitation wavelength of $360 \mathrm{~nm}$ and emission wavelength of $460 \mathrm{~nm}$. The fluorescence intensity of the AMC released is linearly proportional to the enzyme activity in the original serum sample.

Procedure:First, 70ul of assay buffer (composed of $1 \mathrm{mM}$ EDTA, $50 \mathrm{ug} / \mathrm{ml}$ heparin, $150 \mathrm{mM} \mathrm{NaCl}$, tris buffer at $\mathrm{pH} 7.4$ and $1.5 \%$ dimethylsulphoxide (DMSO, used as solvent for substrate solution), and $5 \mathrm{ul}$ of stock substrate (Ac-Orn-PheArg-AMC, obtained from Sigma Aldrich, plc) was added to each well of 96-well microtiter plates [30] and mixed. Then, $25 \mathrm{ul}$ of serum sample was added and the whole $100 \mathrm{ul}$ sample mixtures in each well were mixed and incubated at room temperature for 10 minutes. Fluorescence was then measured using a microtiter plate fluorimeter (excitation wavelength $360 \mathrm{~nm}$, emission wavelength $460 \mathrm{~nm}$ ). The process was automated and the fluorescence converted to enzyme activities measured as $\mathrm{pM} / \mathrm{sec}$ of AMC released, which is equivalent to $\mathrm{pM} / \mathrm{sec}$ of substrate digested.

\subsection{Statistical Analysis}

All the data collected were coded and entered into Excel data sheet on computer. Double entry method was applied to 
preserve data quality. Analysis was done using the Statistical Package for Social Sciences (SPSS) version 20. Results were analyzed statistically using descriptive statistics, Paired Samples T-test, Wilcoxon Signed Rank test and Spearman's Rho test. All the analyzed data were expressed as Median and Mean $\pm \mathrm{SD}$. The minimum level of statistical significance was set at p-value $<0.05$.

\subsection{Definitions}

a. Chronic phase of CML: Early stage of CML in which almost all leukemic cells resemble mature blood cells.

b. Accelerated/ blast phase of CML: Advanced stage of CML in which almost all leukemic cells resemble immature (blast) blood cells.

c. De novo AML:AML acquired by patients from treatment given for other cancers.

d. Secondary AML: AML acquired by patients from chemotherapies given previously for the treatment of other hematological malignancies or solid tumors.

e. Morphologically confirmed CML/AML patients: CML or AML patients diagnosed based on bone marrow and peripheral blood morphologies.

f. Picomolar per second ( $\mathrm{pM} / \mathrm{sec})$ : Stands for increase in the concentration of flourophore product (AMC) in picomolar per second $(\mathrm{pM} / \mathrm{sec})$ or decrease in the concentration of substrate in picomolar per second ( $\mathrm{pM} / \mathrm{sec})$.

g. Presumed healthy controls: Individuals with no major known chronic medical conditions, not taking any medications chronically, never-smokers, occasional or never drinkers of alcoholic beverages.

\section{Results}

\subsection{Age-Sex Distribution of the Study Participants}

Table 1 shows the age/sex distribution of study participants. Sixty morphologically confirmed adult CML patients between 18 and 70 years old; 24 morphologically confirmed AML patients between 19 and 75 years old; and 35 presumed healthy individuals between 26 and 66 years old participated in this study. The mean age for CML patients was $39.1 \pm 15.2$ years; for AML patients was $35.7 \pm 14.1$ years old; and for healthy individuals was $38.5 \pm 10.1$. Therefore, healthy and leukemia patients were age-matched (Paired Samples T-test, \% CI= 95, p-value $=0.00$ ). However, there were more females $(68.6 \%)$ than males $(31.4 \%)$ in the healthy group, but more males than females in both AML and CML patients, so leukemic patients were not well sexmatched with the healthy controls (Paired Samples T-test, \% $\mathrm{CI}=95$, p-value $=0.38$ ).

Table 1.Age-sex distribution of study participants at Hematology-Oncology Clinic of TASH, Addis Ababa, Ethiopia, 2016.

\begin{tabular}{|c|c|c|c|c|}
\hline \multirow{2}{*}{ Participants } & \multicolumn{3}{|l|}{ Sex } & \multirow{2}{*}{ Age $($ Mean \pm SD) years } \\
\hline & Males N (\%) & Females N (\%) & Total N (\%) & \\
\hline CML patients & $39(65 \%)$ & $21(35 \%)$ & $60(100 \%)$ & $39.1 \pm 15.2$ \\
\hline AML patients & $15(62.5 \%)$ & $9(37.5 \%)$ & $24(100 \%)$ & $35.7 \pm 14.1$ \\
\hline Healthy controls & $11(31.4 \%)$ & $24(68.6 \%)$ & $35(100 \%)$ & $385+10$ \\
\hline Total (\%) & $65(54.6 \%)$ & $54(45.4 \%)$ & $119(100 \%)$ & 50.0110 .1 \\
\hline P- Value & \multicolumn{3}{|c|}{$\mathrm{P}=0.38(\mathrm{P}>0.05)$} & $\mathrm{P}=0.00(\mathrm{P}<0.05)$ \\
\hline
\end{tabular}

\subsection{Results of Serum Levels of Tryptase Activity}

The mean \pm SD of serum levels of tryptase activity of study participants (assayed as Ac-Orn-Phe-Arg-AMC hydrolysis) was $291.5 \pm 125.9 \mathrm{pM} / \mathrm{sec}$ for CML patients, $357.9 \pm 220.2 \mathrm{pM} / \mathrm{sec}$ for AML patients and $237.2 \pm 42.8$ $\mathrm{pM} / \mathrm{sec}$ for healthy controls. Paired Samples T-test was performed to see if there was significant difference in the mean serum tryptase levels between AML patients, CML patients and healthy controls. It was found that the mean level of serum tryptase activity (in $\mathrm{pM} / \mathrm{sec}$ ) was significantly lower in healthy controls than in CML patients and AML patients (Paired Samples T-test, \% CI $=95$, p-value $=0.00$ ) (Table 2).

Wilcoxan Signed Rank test was performed to see if there was significant difference in the median serum tryptase levels between AML patients, CML patients and healthy controls. It was found that the median level of serum tryptase activity (in $\mathrm{pM} / \mathrm{sec})$ was significantly lower in healthy controls $(241.5$ $\mathrm{pM} / \mathrm{sec}$ ) than in CML patients (266 pM/sec) and $\mathrm{AML}$ patients $(273.5 \mathrm{pM} / \mathrm{sec})$ (Wilcoxan Signed Rank test, \% CI = 95, p-value $=0.00)($ Table 2$)$.

Table 2.Levels of serum tryptase activity (assessed by Ac-Orn-Phe-Arg-AMC hydrolysis) of study participantsat Hematology-Oncology Clinic of TASH, Addis Ababa, Ethiopia, 2016.

\begin{tabular}{llll}
\hline \multirow{3}{*}{ Stasticalparameters } & \multicolumn{2}{l}{ Serum Levels of Tryptase activity (in pM/sec) } & AML patients \\
\cline { 2 - 4 } & Healthy Controls & CML patients & P-Value \\
\cline { 2 - 4 } & Ac-Orn-Phe-Arg-AMC & Ac-Orn-Phe-Arg-AMC & Ac-Orn-Phe-Arg-AMC \\
\hline Mean \pm SD & $237.2 \pm 42.8$ & $291.5 \pm 125.9$ & $357.9 \pm 220.2$ \\
Median & 241.5 & 266 & 273.5 \\
Range & $132-298$ & $122-701$ & $131-901$ \\
\hline
\end{tabular}


Except, for one abnormally higher value of $599 \mathrm{pM} / \mathrm{sec}$ (outlier), the maximum serum level of tryptase activity for healthy controls (assayed as Ac-Orn-Phe-Arg-AMC hydrolysis) was $298 \mathrm{pM} / \mathrm{sec}$.Ignoring the outlier (599 $\mathrm{pM} / \mathrm{sec}$ ), therefore, $298 \mathrm{pM} / \mathrm{sec}$ was used as a normal upper limit for serum levels of tryptase activity in this study. Accordingly, 10/24 (41.7\%) AML patients had elevated tryptase levels, whereas 18/60 (30\%) CML patients had elevated serum tryptase levels. As already stated, only $1 / 35$ $(2.9 \%)$ of healthy controls had elevated serum tryptase. However, in 14/24 (58.3\%) AML patients and 42/60 (70 \%) CML patients the serum tryptase level was considered to be in the normal range (below $298 \mathrm{pM} / \mathrm{sec}$; Figure 5).

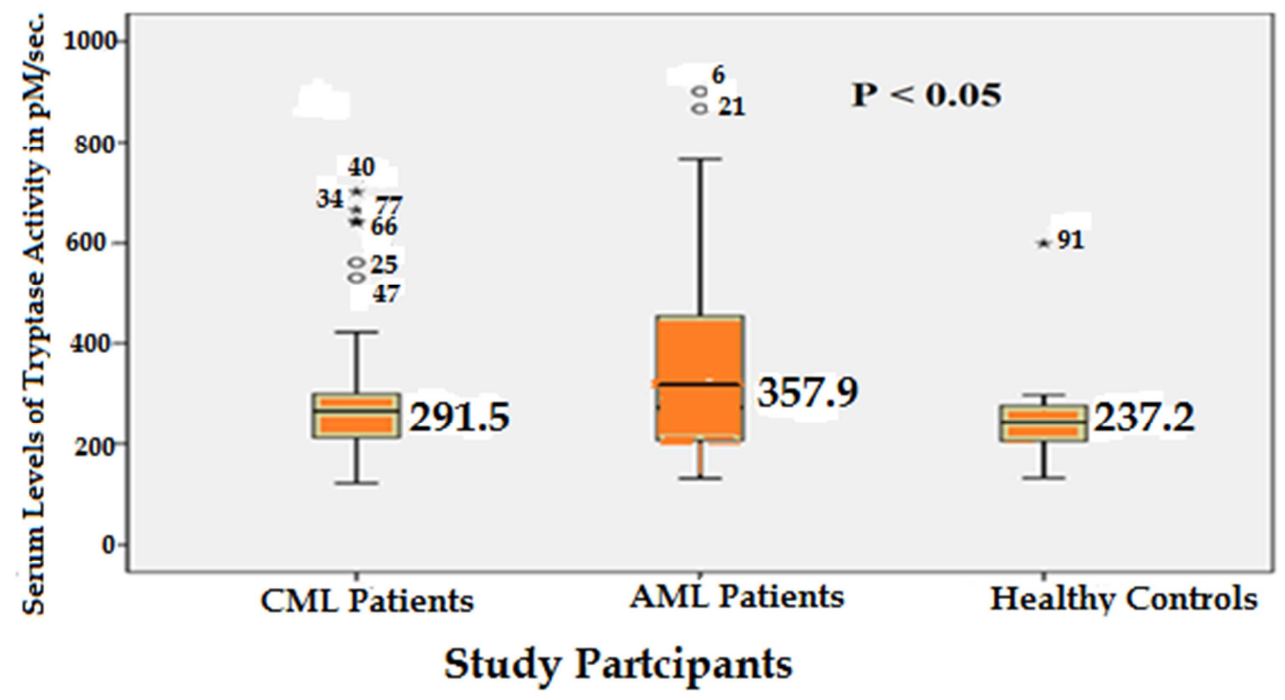

Figure 5. Serum levels of tryptase activity (assessed by Ac-Orn-Phe-Arg-AMC hydrolysis) of study participantsat Hematology-Oncology Clinic of TASH, Addis Ababa, Ethiopia, 2016.

$6,21^{\circ}$ indicate outliers of AML samples and 25, $47^{\circ}$ indicate outliers of CML samples, and 34, 40, 77* indicate abnormally elevated outlier values of CML samples and $91^{*}$ indicates abnormally elevated outlier values of healthy controls in terms of sample identification codes $(6,21,25$, 47, 34, 40, 77 and 91).

\subsubsection{Serum Levels of Tryptase Activity and Classes of AML}

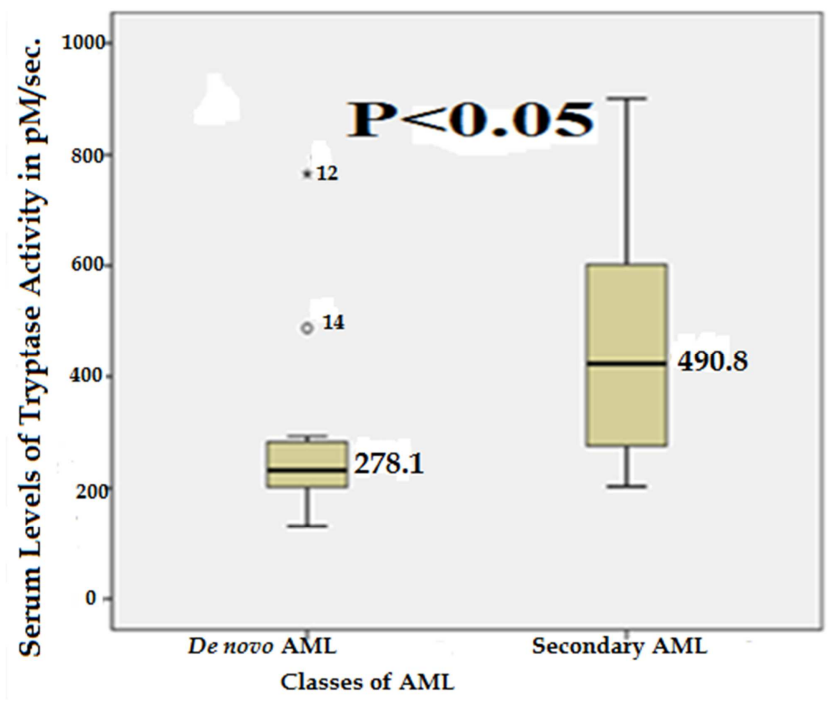

Figure 6. Serum levels of tryptase (assayed as AC-Orn-Phe-Arg-AMC hydrolysis) activity of de novo and secondary AML patients at HematologyOncology Clinic of TASH, Addis Ababa, Ethiopia, 2016.
In the present study, the serum levels of tryptase activity of AML patients with respect to two etiological classes: de novo AML and secondary AML, were assessed and compared to each other. The mean \pm SD and median level of serum tryptase activity (in $\mathrm{pM} / \mathrm{sec}$ ) (assessed by Ac-Orn-Phe-ArgAMC hydrolysis) was determined for each class of AML. The mean \pm SD and median level of serum tryptase activity was higher in secondary AML patients than in patients with de novo $\mathrm{AML}$ (mean $\pm \mathrm{SD}=490.8 \pm 253.6$, median $=423$ versus $278.1 \pm 157.4,232$ (Paired samples T-tests and Wilcoxan Rank Test, $\% \mathrm{CI}=95$, p-value $<0.05$; Figure 6).

$14^{\circ}$ indicates outlier value of de novo AML samples and $12 *$ indicates abnormally elevated outlier value of de novo AML samples in terms of sample identification codes (12 and 14).

\subsubsection{Serum Levels of Tryptase Activity and Stage of CML}

Comparison of the serum levels of tryptase in different stages of CML revealed higher mean $\pm \mathrm{SD}$ and median difference in CML patients in the chronic (CP) phase than CML patients in the accelerated/blast (AP/BP) phase. The mean \pm SD and median level of serum tryptase activity (in $\mathrm{pM} / \mathrm{sec}$ ) (assessed by Ac-Orn-Phe-Arg-AMC digestion) was determined for each phases of CML and it was found that the mean \pm SD and median level of serum tryptase activity for CML patients in the chronic phase (CP) was higher than that of those in the accelerated /blast (AP/BP) phase (mean \pm SD $=298.5 \pm 154.5$ versus $287.9 \pm 110.9$ and median $=276.5$ versus 234.5(Paired samples T-tests and Wilcoxan Rank Test, $\% \mathrm{CI}=95$, $\mathrm{p}$-value $<0.05$; Figure 7 ). 


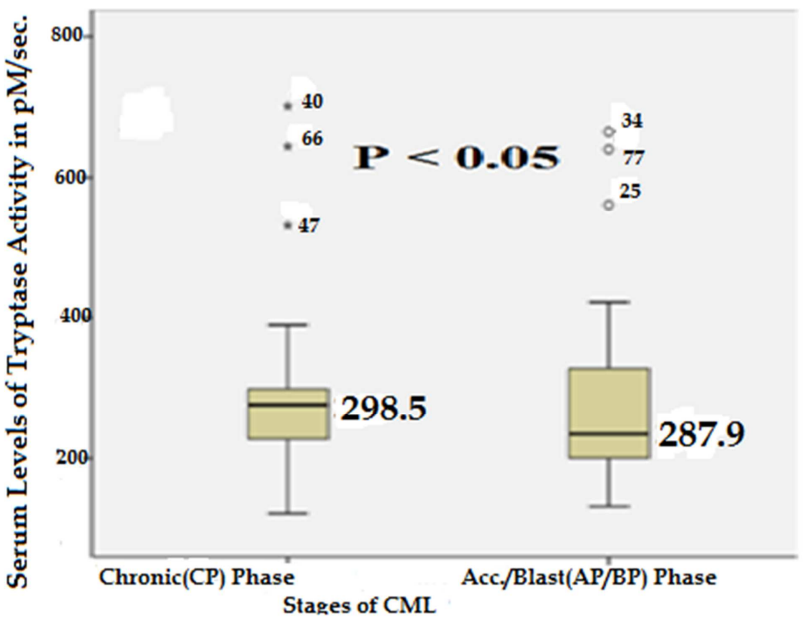

Figure 7. Serum levels of tryptase (assayed as Ac-Orn-Phe-Arg-AMC hydrolysis) activity of $C M L$ patients in $C P$ and $A P / B P$ stagesat HematologyOncology Clinic of TASH, Addis Ababa, Ethiopia, 2016.

25, 34 and $77^{\circ}$ indicate outlier values of AP/BP CML samples and 40, 47 and $66^{*}$ indicates abnormally elevated outlier values of CML samples on the CP side in terms of sample identification codes $(25,34,40,47,66$ and 77$)$.

In summary, the mean $\pm \mathrm{SD}$ and median values of serum levels of tryptase activity of CML and AML patients were higher when compared with those of healthy controls. The mean \pm SD and median values of serum levels of tryptase activity of AML patients were higher than those of CML patients.

Except for one very high value of $599 \mathrm{pM} / \mathrm{sec}$ (outlier), the maximum serum level of tryptase activity value record for healthy controls was $298 \mathrm{pM} / \mathrm{sec}$.Ten out of 24 AML patients and 18/60 CML patients had elevated serum levels of tryptase activity (above $298 \mathrm{pM} / \mathrm{sec}$ of healthy controls). Furthermore, a higher mean \pm SD and median value of serum tryptase levels were found in secondary AML patients compared with de novo AML patients and CML patients in the $\mathrm{CP}$ stage than AP/BP stage.

\section{Discussion}

In the present study it was found that the median and mean $\pm \mathrm{SD}$ values of serum levels of tryptase assessed by hydrolysis of Ac-Orn-Phe-Arg-AMC were higher in patients with AML and CML when compared with values of serum tryptase levels in healthy controls with statistical significance of $\mathrm{P}$-value $<0.05$. In agreement with this finding, other studies also showed higher serum levels of tryptase among AML and CML patients [4], [25], [27], [28], pancreatic cancer patients [29], gastric cancer patients [3], colorectal cancer [2] and breast cancer [15] patients, than in healthy controls.

The higher mean and median value of serum levels of tryptase activity in AML and CML patients of this study did not occur in most or all patients, but in a subset of patients with AML and CML. Most patients had normal serum levels of tryptase activity which is consistent with findings from other studies.For example, one study reported normal tryptase level in the serum of $62 \%$ of AML patients and $66 \%$ of CML patients [27]. Similarly, one other studyreported normal tryptase levels in the serum of $61 \%$ of de novo AML patients and $56 \%$ of secondary AML patients [28].

One possible explanation for the elevation of serum tryptase activity in only a subset of leukemia patients could be genetic variations that predispose to high or low tryptase levels among CML and AML patients. Another possible explanation is that there are fluctuations in the levels of tryptase in AML and CML patients during the course of their disease and that some patients with low levels of serum tryptase activity might, at other times, have elevated levels. This idea could be tested by following serum levels of tryptase activity during the time course of the leukemia. In addition, it may be that serum level of tryptase activity is a marker for a subset of leukemia or for the degree of aggressiveness of AML/CML.

Generally, findings support two hypotheses for serum levels of tryptase as biomarkersin cancer patients: Mast cellderived biomarker hypothesis and tumour cell-derived biomarker hypothesis. The former hypothesis was supported by studies done on the colorectal cancer [2], gastric cancer [3], breast cancer [15] and pancreatic cancer [29]. The later hypothesis was supported by recent findings suggesting the production of the significant amount of tryptase by the AML blasts in patients with AML and immature CML basophiles in patients with CML [25], [26].

Moreover, a study done among patients with hematological malignancies revealed that serum tryptase levels were higher in almost all patients with myeloid neoplasm compared with patients of lymphoid neoplasm [27].In the same study, $38 \%$ of AML patients had an elevated serum tryptase level and $34 \%$ CML patients had an elevated serum tryptase level [27]. In one other study of 108 patients with de novo AML and 25 patients with secondary AML elevated serum tryptase levels was detected in $39 \%$ of patients with de novo AML and $44 \%$ of those with secondary AML [28].In this study, the proportion of AML and CML patients with elevated serum tryptase level is similar to the proportions indicated by other studies [27], [28], i.e. about $41.6 \%$ of AML and $30 \%$ of CML patients of present study had elevated serum tryptase levels.

Almost all studies done previously, those that support a mast cell-derived hypothesis for serum tryptase in cancer patients [2], [3], [15], [29] or those that support a tumorderived hypothesis for serum tryptase in AML and CML patients [4], [25], [26], [27], [28],determined the serum tryptase levels by using quantitative tryptase immunoassay. This assay detects only the amount of protein (tryptase) in the serum but not the activity of the enzyme (tryptase). This study, on the other hand, measured the enzyme activity, not quantitative tryptase protein that is measured using immunoassay. It would be interesting to compare immunoassay with enzyme assay results in these patients.

Though Ac-Orn-Phe-Arg-AMC used in this study is especially hydrolyzed by tryptase, other proteases in serum 
might also digest this substrate. Therefore, a proportion of the elevated activity of Ac-Orn-Phe-Arg-AMC hydrolysis might not be due to tryptase, and might be due to the presence of other non-tryptase protease(s) in the serum that digest the substrate Ac-Orn-Phe-Arg-AMC in the same manner as tryptase does.Other substrates, including substrates with greater specificity for tryptase, might help solve this problem, as immunoassay for tryptase and fractionation of serum samples by chromatographic methods might.

Several studies suggested the important roles of tryptase in many pathological conditions such as allergic airway diseases, chronic renal disease, rheumatoid arthritis, and tumor (cancer) angiogenesis ([5], [8], [11], [21], [23]. For example, studies indicated increased mast cell tryptase secretion in nasal lavage fluid of grass pollen allergic patients [21] and other studies suggested increased serum or bronchoalveolar lavage mast cell tryptase level in asthmatics after endobronchial allergen challenge [5], [10], [21].One other study reported elevated serum tryptase level in chronic kidney disease patients [23].

In this study one highly elevated serum tryptase value (599 $\mathrm{pM} / \mathrm{sec}$ ) among healthy controls (Figure 5) was found. There are many possibilities for this higher value of serum tryptase activity recorded in one isolated case of healthy controls. One possibility might be measurement or experimental errors e.g. contamination of serum sample with some bacterial proteases that could digest Ac-Orn-Phe-Arg-AMC. It is also possible that a serum sample mix-up occurred. To be certain, the procedure could be repeated with the same serum sample to see if the elevated serum tryptase value is reproduced. Another possibility might be that the individual had an allergic condition or reaction that was asymptomatic[5], [11], [21] or other conditions namely, mastocytosis, rheumatoid arthritis that might raise the serum levels of tryptase activity [5], [8], [10], [21]. It could also be that the healthy control with the high tryptase activity simply had a genetic predisposition for having such elevated serum tryptase activity without having any pathological condition.

In the present study, it was found that the mean \pm SD and median value of serum tryptase level assessed in terms of digestion of Ac-Orn-Phe-Arg-AMC was higher in secondary AML patients than in patients with de novo AML with statistical significance of $\mathrm{P}$-value $<0.05$. In agreement with this finding, other studies (39\% versus $44 \%$ [28], $36.3 \%$ versus $45 \%$ [4]) also showed higher serum levels of tryptase in patients with secondary AML than de novo AML.

It was shown in one studythat patients with advanced (accelerated and blast) phase CML more often possess elevated serum-tryptase levels than patients with chronic phase CML [26]. In this study, however, it was found that the mean $\pm \mathrm{SD}$ and median value of serum tryptase level assessed in terms of digestion of Ac-Orn-Phe-Arg-AMC for CML patients in $\mathrm{CP}$ was higher than that of those in AP/BP (Figure 7).The reason behind this disagreement is unclear, but it might be due to the difference in tryptase assay method used or the presence of outlier values of CML samples in the
CP than AP/BP side (Figure 7) in this study.

\section{Conclusions}

In this study, elevated mean \pm SD and median levels of serum tryptase activity were seen in AML and CML patients when compared with healthy controls. However, the elevation was due to elevation in a subset of patients.

Among leukemic patients included in this study, mean and median serum level of tryptase activity was higher in AML patients than CML patients; in patients with secondary AML than de novo AML; and in CML patients in the chronic phase (CP) than in the accelerated/ blast phase (AP/BP).

\section{References}

[1] American Cancer Society (2015). Leukemia-Acute Myeloid (Myelogenous). Cancer Facts \& Figures. Atlanta, Ga: American Cancer Society; 1-60.

[2] Ammendola M., Leporini C., Marech I.,Gadaleta D. C., Scognamillo G.,Sacco R., Sammarco G.,De Sarro G., Russo E., and Ranieri G. (2014a). Targeting Mast Cells Tryptase in Tumor Microenvironment:A Potential Antiangiogenetic Strategy.Review Article: BioMed Research International, http://dx.doi.org/10.1155/2014/154702; 1-16.

[3] Ammendola M., Sacco R., Sammarco G., Donato G., Montemurro S., Ruggieri E., Patruno R., Marech I., Cariello M.,Vacca A., Gadaleta D. C. and Ranieri G. (2014b). Correlation between Serum Tryptase, Mast Cells Positive to Tryptase and Microvascular Density in Colo-Rectal Cancer Patients: Possible Biological-Clinical Significance. PLoS ONE/www.plosone.org; 9(6): e99512.

[4] Cairoli R., Ripamonti B. C., Beghini A., Granata S., Grillo G., Brioschi M., Nadali G., Viola A., Cattaneo C., Inropido L., Ravelli E., Bertani G., Pezzetti L., Nichelatti M., Marocchi A., Rossi G., Pizzolo G., Ferrara F., Nosari M. A., Morra E. (2009). Total serum tryptase: A predictive marker for KIT mutation in acute myeloid leukemia. Leukemia Research; 33: $1282-1284$.

[5] Caughey H.G., Raymond W. W., Blount L. J., Hau T. W.,Pallaoro M., Wolters J. P., and Verghese M. G. (2000). Characterization of Human g-Tryptases, Novel Members ofthe Chromosome 16p Mast Cell Tryptase and Prostasin GeneFamilies. The Journal of Immunology; 164: 6566-6575.

[6] Davies J.N.P. (1973). Childhood tumors. In: Templeton, A.C., ed., Tumors in a TropicalCountry (Recent Results in Cancer Research No. 41), Berlin, Springer Verlag.

[7] Gocek E. and Marcinkowska E. (2011). Differentiation Therapy of Acute Myeloid Leukemia. Review; 3: 2402-2420.

[8] Guo Y., Wu Q., Ni B., Mou Z., Jiang Q., Cao Y., Dong H. and Wu Y. (2013). Tryptase is a candidate autoantigen in rheumatoid arthritis. The Journal of cells, molecules,systems and technologies; 142, 67-77.

[9] Ibrahim E. and Osman I. (2011). Myeloid Leukemia: A Molecular Focus on Etiology and Risk Within Africa, Myeloid Leukemia Basic Mechanisms of Leukemogenesis, Steffen Koschmieder (Ed.), ISBN: 978-953-307-789-5. In Tech, Available from: $\mathrm{http}: / /$ www.intechopen.com/books/myeloid-leukemia. 
[10] Itoh Y., Sendo T., and Oishi R. (2005). Physiology and Pathophysiology of Proteinase Activated Receptors (PARs): Role of Tryptase/PAR-2 in Vascular Endothelial Barrier Function.Journal of Pharmacological Sciences; 97:14-19.

[11] Junior A. D., Santana C. A.,Marcelino da Silva Z. E., Oliver C., and Jamur C. M.(2015). The Role of Mast Cell Specific Chymasesand Tryptases in Tumor Angiogenesis. Review Article: BioMed Research International;1-13.

[12] Kasili E. G. (1990). Childhood Leukemia: Is it a Problem in Tropical Africa? Leukemia \&Lymphoma, Vol. 1, No. 3-4, 187193.

[13] King T.G.(2014). Serum Tryptase: Reference range, Interpretation, collection and panel. Madcaps Drugs and Disease; $1-10$.

[14] Lockwood W. (2015). Leukemia: AML, CML, ALL and CLL. www.RN.ORG. (C2015 RN.ORG®, S.A..RN.ORG®, LLC. ISBN: 678-693-1-32.

[15] Marech I., Ammendola M., Gadaleta C., Zizzo N., Oakley C., Gadaleta D. C., and Ranieri G. (2014). Possible biological and translational significance of mastcells density in colorectal cancer. World JGastroenterol; 20(27): 8910-8920.

[16] Mori S.,Itoh Y., Shinohata R., Sendo T., Oishi R., and Nishibori M. (2003). Nafamostat Mesilate Is an Extremely Potent Inhibitor of Human Tryptase. Journal of Pharmacological Sciences; 92:420-23.

[17] National Cancer Institute, (2001). Genes and Disease: blood and lymph diseases. Available at: Cancer.gov (http://www.cancer.gov/cancerinfo/wyntk/leukemia.)

[18] Nwannadi, O. Alao, G. Bazuaye, M. Nwagu \& M. Borke (2011). Clinical and Laboratory Characteristics of Patients with Leukaemia in South-South Nigeria. The Internet Journal of Oncology; 7 (2).

[19] Obama, M.T., L. Zekeng, P.K. Ketchiozo, M.B. Owono, B.T. Kouam, \& J. Mbede. (1995).Childhood leukemia is still a deadly disease in Yaounde, Cameroon: a report of 14cases. Pediatr Hematol Oncol 12: 301-304.

[20] Roboz J.G. (2011). Novel Approaches to the Treatment of Acute MyeloidLeukemia.Acute Myeloid Leukemia in the Age of Genomics; 43-50.

[21] Sendo T.,Itoh Y., Goromaru T., Sumimura T., Saito M., Aki K., Yano T., and Oishi R. (2003). A potent tryptase inhibitor nafamostat mesilate dramatically suppressed pulmonary dysfunction induced in rats by a radiographic contrast medium. British Journal of Pharmacology; 138: 959-967.
[22] Shamebo M. (1990). Leukaemia in adult Ethiopians. Ethiop Med J, 28(1): 31-7.

[23] Sirvent A., González C., Enríquez R., Fernández J., Millán I., Barber X. and Amorós F. (2010). Serum tryptase levels and markers of renal dysfunction in a population with chronic kidney disease JNEPHROL; 23(03): 282-290.

[24] Sommerhoff P. C., Bode W., Pereira B. J., Stubbs T.M., Rzebecher J., Piechottka P. G., Matschiner G., and Bergner A. (1999).The structure of the human BII-tryptase tetramer: Fo (u) r betteror worse. Proc. Natl. Acad. Sci. USA; 96: 10984 10991.

[25] Sperr R. W., Mitterbauer M., Mitterbauer G., Kundi M., Jager U., Lechner K., and Valent P. (2005). Quantization of Minimal Residual Disease in Acute Myeloid Leukemia by Tryptase Monitoring Identifies a Group of Patients with a High Risk of Relapse. Clin Cancer Res. Imaging, Diagnosis, Prognosis; 11(18): 6536-43.

[26] Sperr R. W., Pfeiffer T., Hoermann G., Herndlhofer S., Mannhalter C.,Kundi M., and Valent P. (2015a).Serumtryptase at diagnosis: a novel biomarker improving prognostication in Ph+ CML. Am J Cancer Res; 5(1):354-362.

[27] Sperr R.W., El-Samahi A., Kundi M., Girschikofsky M., Winkler S., Lutz D., Endler G., Rumpold H., Agis H., Sillaber C., Jager U. and Valent P.(2009). Elevated tryptase levels selectively cluster in myeloid neoplasms: a novel diagnostic approach and screen marker in clinical haematology. European Journal of Clinical investigation; 39(10): 914-923.

[28] Sperr R.W., Jordan H.J., Baghestanian M., Kiener P. H., Samorapoompichit P.,HauswirthA. H., Schernthaner H.G., Chott A., Natter S., Kraft D., Valenta R., Schwartz B. L., Geissler K., Lechner K., and Valent P. (2015b). Expression of mast cell tryptase by myeloblasts in a group of patients with acute myeloid leukemia. Neoplasia: www.bloodjournal.org; 98(7): 2200-2209.

[29] Strouch M., Cheon E., Salabat M., KrantzS., Gounaris E., Melstrom L., Dangi-Garimella S., WangE.,Munshi H., KhazaieK., and BentremD. (2011). Crosstalk between mast cells and pancreatic cancer cellscontributes to pancreatic tumor progression. Clin Cancer Res.; 16(8): 2257-2265. doi:10.1158/1078-0432.CCR-09-1230.

[30] Wysocka M. and Lesner A. (2013). Future of Protease Activity Assays. Current Pharmaceutical Design; 19(6): 1062-1067.

[31] Zhang G. (2006). Protease Assays. Assay Guidance Manual; NBK9: pp. 1-14. 